\title{
GROWTH SERIES OF SOME WREATH PRODUCTS
}

\author{
WALTER PARRY
}

\begin{abstract}
The growth series of certain finitely generated groups which are wreath products are investigated. These growth series are intimately related to the traveling salesman problem on certain graphs. A large class of these growth series is shown to consist of irrational algebraic functions.
\end{abstract}

\section{INTRODUCTION}

Let $\Gamma$ be a group with a finite generating set $S$. Define the $S$-length $\|g\|_{S}$ of an element $g$ in $\Gamma$ to be the least nonnegative integer $n$ such that $g$ can be expressed as a product of $n$ elements from $S \cup S^{-1}$. For every nonnegative integer $n$ let $a_{n}$ be the number of elements in $\Gamma$ with $S$-length $n$. In particular, $a_{0}=1$ (the identity element), and $a_{1}$ is the number of nonidentity elements in $S \cup S^{-1}$. The growth series of the pair $(\Gamma, S)$ is by definition

$$
f_{\Gamma}(x)=\sum_{n=0}^{\infty} a_{n} x^{n}
$$

Since only one generating set will be associated with each group below, the generating set associated with $f_{\Gamma}(x)$ will be obvious.

Let $H$ be a group with a finite generating set $S_{H}$. These will be fixed for the rest of the paper. The Cayley graph of the pair $\left(H, S_{H}\right)$ is, as usual, the directed graph whose vertices are the elements of $H$ and there is an edge from a vertex $h_{1}$ to a vertex $h_{2}$ if and only if $h_{2}=h_{1} h$ for some $h$ in $S_{H} \cup S_{H}^{-1}$. In particular, the edge from $h_{1}$ to $h_{2}$ has an opposite edge from $h_{2}$ to $h_{1}$. Let $C$ be the graph gotten from the Cayley graph of $\left(H, S_{H}\right)$ simply by identifying opposite edges. In other words, $C$ might be called the undirected Cayley graph of $\left(H, S_{H}\right)$.

Let $K$ also be a group with a finite generating set $S_{K}$. These will also be fixed for the rest of the paper. It is possible to form what might be called a restricted direct product group $P$, which consists of all functions $p$ from the vertices of $C$ to $K$ such that there are only finitely many vertices $v$ in $C$ with $p(v) \neq 1$. This is a subgroup of the direct product group whose elements consist of all functions from the vertex set of $C$ to $K$. The group $P$ admits $H$ as a group of automorphisms by means of the action of $H$ on $C$. The resulting semidirect product $P \rtimes H$ is the restricted wreath product $K \backslash H$.

Received by the editors March 10, 1990.

1980 Mathematics Subject Classification (1985 Revision). Primary 20F32, 05C25; Secondary $20 \mathrm{E} 22$. 
Set $G=K \imath H$. It is possible to identify $H$ and $P$ with subgroups of $G$ in a natural way. Suppose that the identity element of $H$ projects to the vertex $v_{0}$ in $C$. Identify $K$ with the subgroup of $P$ consisting of those functions $p$ such that $p(v)=1$ if $v \neq v_{0}$. Having done this, $S_{H}$ and $S_{K}$ lie in $G$. Set $S_{G}=S_{H} \cup S_{K}$. This set generates $G$.

In $\S 1$ it will be shown that the problem of determining the $S_{G}$-lengths of the elements of $G$ is intimately related to the traveling salesman problem in $C$. The precise result is in Theorem 1.2. It is for this reason that Jim Cannon calls these groups lamplighter groups. (Brief explanation: a lamplighter lives in a town whose street map is $C$ and walks about the town lighting various lamps (elements of $K$, especially when $K=\mathbb{Z} / 2 \mathbb{Z}$ ).)

After $\S 1$ it will be assumed that $C$ is a tree. In $\S 3.3$ of [6] it is shown that this together with the assumption that $H$ acts without inversions implies that $H$ is a free group. However, the elements of $H$ are allowed to act by inversion here. It follows that $H$ is a free product of groups isomorphic to either $\mathbb{Z}$ or $\mathbb{Z} / 2 \mathbb{Z}$. The valence of the vertices of $C$ will be denoted by $m$ (which can be any nonnegative integer).

Section 2 is devoted to obtaining an interesting expression for $f_{G}(x)$ in terms of $f_{K}(x)$ and a generating function $F_{C}(x, y)$ of two variables which is associated to certain finite subtrees of $C$. This result is in Theorem 2.6. I know very little about generalizing Theorem 2.6 to the case in which $C$ is not a tree. In particular, what about the case in which $H=\mathbb{Z}^{2}$ and $S_{H}$ is the standard basis?

Section 3 investigates to what extent $f_{G}(x)$ is a rational function. Lemma 3.1 shows that $F_{C}(x, y)$ is algebraic over the field generated over $\mathbb{Q}$ by $x$ and $y$. Corollary 3.2 shows that the power series $F(x)$ in Theorem 2.6 is algebraic over the field generated over $\mathbb{Q}$ by $x$ and $f_{K}(x)$. It is easy to see that the equation in Corollary 3.2 can be used to compute the coefficients of $F(x)$ recursively. Thus to compute $f_{G}(x)$ for $m \geq 1$ given $f_{K}(x)$, one can use the equation in Corollary 3.2 to compute $F(x)$ and then Theorem 2.6 to compute $f_{G}(x)$. Moreover if $m \leq 2$, then Corollary 3.3 gives $f_{G}(x)$ as a rational function of $x$ and $f_{K}(x)$. Thus in this case $f_{G}(x)$ is a rational function if $f_{K}(x)$ is a rational function. However, if $m>2$ and $K \neq 1$, then although $f_{G}(x)$ is algebraic over the field generated over $\mathbb{Q}$ by $x$ and $f_{K}(x)$, it is not contained in this field by Theorem 3.7. Thus if $m>2, K \neq 1$ and $f_{K}(x)$ is rational, then $f_{G}(x)$ is an irrational algebraic function. As far as I know the only examples of groups with irrational growth series previously known are finitely presented groups with unsolvable word problems [2] and the examples presented in [4]. None of these power series is algebraic.

Line $(0.1)$ will be needed later, and so it seems appropriate to now return to the setting of the first paragraph and discuss the radius of convergence of $f_{\Gamma}(x)$. For every nonnegative integer $n$ let $b_{n}$ be the number of elements in $\Gamma$ with $S$-length at most $n$. Clearly, $b_{n}=\sum_{i=0}^{n} a_{i}$. The remark which begins on p. 1 of [5] shows that the limit $\lim _{n \rightarrow \infty} b_{n}^{1 / n}$ exists. It is not difficult to see that $\lim _{n \rightarrow \infty} b_{n}^{1 / n}=\lim _{n \rightarrow \infty} a_{n}^{1 / n}$ if $\Gamma$ is an infinite group, and so in this case $\lim _{n \rightarrow \infty} b_{n}^{1 / n}=1 / R$, where $R$ is the radius of convergence of $f_{\Gamma}(x)$. The aforementioned remark in [5] contains for each fixed $t$ the inequality $\lim \sup \gamma(s)^{1 / s} \leq \gamma(t)^{1 / t}$. This can be rewritten in the present notation as $1 / R \leq b_{n}^{1 / n}$, hence $b_{n} R^{n} \geq 1$. This easily proves the following statement. 
If $R$ is the radius of convergence of the growth series of a group with respect to a finite generating set, then the values of the growth series approach $\infty$ as the variable increases to $R$.

Section 4 deals with the radius of convergence of $f_{G}(x)$. Corollary 3.3 easily shows for $m=0,1$ that the radius of convergence of $f_{G}(x)$ equals the radius of convergence of $f_{K}(x)$. Thus in $\S 4$ it will be assumed that $m \geq 2$. Theorem 4.1 describes the radius of convergence of $f_{G}(x)$ for $m \geq 2$.

The paper concludes with $\S 5$ in which some examples are discussed.

It is a pleasure for me to acknowledge here the friendly assistance of Jim Cannon and Bill Floyd in the course of writing this paper.

\section{Walks in $C$}

It is appropriate at this point to explicitly describe the conjugation action of $H$ on $P$. If $p$ lies in $P$ and $h$ lies in $H$, then the value of $h p h^{-1}$ at $h v$ is the value of $p$ at $v$ for every vertex $v$ of $C$.

Now suppose given an element $g$ in $G$, and consider the problem of determining $\|g\|_{S_{G}}$. Suppose that $g$ is given as a product of $\|g\|_{S_{G}}$ elements in $S_{G}=S_{H} \cup S_{K}$. Such a product will be called a minimal representation of $g$. Let the elements $h_{0}, \ldots, h_{q}$ in $H$ (allowing for $h_{0}=1$ or $h_{q}=1$ ) be maximal subproducts of elements in $S_{H}$ and let the elements $k_{1}, \ldots, k_{q}$ in $K$ be maximal subproducts of elements in $S_{K}$ for some $q \geq 0$, so that the given minimal representation of $g$ is a refinement of

$$
g=h_{0} k_{1} h_{1} k_{2} h_{2} \cdots k_{q-1} h_{q-1} k_{q} h_{q} .
$$

In particular,

$$
\|g\|_{S_{G}}=\sum_{i=0}^{q}\left\|h_{i}\right\|_{S_{H}}+\sum_{i=1}^{q}\left\|k_{i}\right\|_{S_{K}} .
$$

Express $g$ as follows.

$$
\begin{aligned}
g= & \left(h_{0} k_{1} h_{0}^{-1}\right)\left(h_{0} h_{1} k_{2} h_{1}^{-1} h_{0}^{-1}\right)\left(h_{0} h_{1} h_{2} k_{3} h_{2}^{-1} h_{1}^{-1} h_{0}^{-1}\right) \\
& \cdots\left(h_{0} \cdots h_{q-1} k_{q} h_{q-1}^{-1} \cdots h_{0}^{-1}\right) h_{0} h_{1} h_{2} \cdots h_{q} .
\end{aligned}
$$

This expression for $g$ can be viewed as taking a walk in $C$ which stops at certain vertices along the way to construct elements of $K$. More precisely, begin at $v_{0}$ and walk a geodesic path to $h_{0} v_{0}$. The distance travelled is $\left\|h_{0}\right\|_{S_{H}}$. At $h_{0} v_{0}$ use $\left\|k_{1}\right\|_{S_{K}}$ elements of $S_{K}$ to construct $k_{1}$. From $h_{0} v_{0}$ walk a geodesic path to $h_{0} h_{1} v_{0}$. The distance travelled is $\left\|h_{1}\right\|_{S_{H}}$. At $h_{0} h_{1} v_{0}$ use $\left\|k_{2}\right\|_{S_{K}}$ elements of $S_{K}$ to construct $k_{2}$. Continue walking in this way until finally stopping at $h_{0} h_{1} h_{2} \cdots h_{q} v_{0}$. In this interpretation, $\|g\|_{S_{G}}$ is the total distance travelled plus the total number of elements from $S_{K}$ used along the way. For the present purposes the term walk will not only include the notion of moving among the vertices, but also the notion of stopping at vertices and constructing elements of $K$. Define a minimal walk for $g$ to be one which corresponds to a minimal representation of $g$ as above.

It will now be shown that there is no advantage to stopping at a vertex more than once. Suppose that there are integers $r, t$ such that $h_{0} \cdots h_{r}=h_{0} \cdots h_{t}$ with $0 \leq r<t<q$ and $h_{0} \cdots h_{r} \neq h_{0} \cdots h_{s}$ for every integer $s$ with $r<s<$ 
$t$. Since $h_{0} \cdots h_{t} k_{t} h_{t}^{-1} \cdots h_{0}^{-1}$ commutes with $h_{0} \cdots h_{s} k_{s} h_{s}^{-1} \cdots h_{0}^{-1}$ for every such $s, g$ is unchanged by deleting $h_{0} \cdots h_{t} k_{t} h_{t}^{-1} \cdots h_{0}^{-1}$ from line (1.1) and replacing $k_{r}$ by $k_{r} k_{t}$. This changes the walk in two ways. First, instead of constructing $k_{r}$ at $h_{0} \cdots h_{r} v_{0}, k_{r} k_{t}$ is constructed. This does not increase the value of $\sum_{i=1}^{q}\left\|k_{i}\right\|_{S_{K}}$ because $\left\|k_{r} k_{t}\right\|_{S_{K}} \leq\left\|k_{r}\right\|_{S_{K}}+\left\|k_{t}\right\|_{S_{K}}$. Second, instead of walking from $h_{0} \cdots h_{t-1} v_{0}$ to $h_{0} \cdots h_{t} v_{0}$ and then to $h_{0} \cdots h_{t+1} v_{0}$, the walk proceeds directly from $h_{0} \cdots h_{t-1} v_{0}$ to $h_{0} \cdots h_{t+1} v_{0}$. The triangle inequality also shows that this does not increase the value of $\sum_{i=0}^{q}\left\|h_{i}\right\|_{S_{H}}$. It is now easy to see that for every element in $G$ there is a minimal walk which stops at every vertex of $C$ at most once.

Now express a given element $g$ in $G$ as $g=p h$ for some $p$ in $P$ and $h$ in $H$. It is clear that every minimal walk for $g$ must pass through every vertex $v$ of $C$ such that $p(v) \neq 1$ and it must end at $h v_{0}$. Consequently, the above proves the following theorem.

Theorem 1.2. Let $g=p h$ be an element in $G$ with $p$ in $P$ and $h$ in $H$. One minimal walk for $g$ is gotten as follows. Take a shortest possible walk in $C$ starting at $v_{0}$, ending at $h v_{0}$ and passing through all vertices $v$ of $C$ for which $p(v) \neq 1$. Stop at the latter vertices $v$ just once and represent $p(v)$ there minimally in terms of $S_{K}$.

It will be convenient to say that the value of a minimal walk for $g$ as in Theorem 1.2 at a vertex $v$ of $C$ is $p(v)$.

\section{Description of $f_{G}(x)$ When $C$ is a tree}

Henceforth it will be assumed that $C$ is a tree. The valence of every vertex of $C$ is the cardinality of $S_{H} \cup S_{H}^{-1}$. Let $m$ denote this valence.

A finite subtree of $C$ can be associated to every finite walk in $C$, namely, the subtree of those vertices and edges traversed in the walk. Call this finite subtree the support of the walk. Define the extreme vertices of a finite tree to be its vertices with valence 1 or 0 (in the case of a tree with just one vertex).

Choose a vertex $v_{1}$ adjacent to $v_{0}$, and let $\mathscr{T}$ be the set of finite subtrees of $C$ which contain $v_{0}$ but contain no vertex adjacent to $v_{0}$ other than $v_{1}$. Then $v_{0}$ is an extreme vertex of such a subtree. Call it the trivial extreme vertex. Let $F_{C}(x, y)$ be the generating function which results from counting the elements of $\mathscr{T}$ according to the numbers of their nonextreme vertices and nontrivial extreme vertices:

$$
F_{C}(x, y)=\sum_{i, j=0}^{\infty} a_{i j} x^{i} y^{j}
$$

where

$$
\begin{aligned}
a_{i j}=\mid\{T \in \mathscr{T}: T \text { has } i \text { nonextreme vertices and } \\
j \text { nontrivial extreme vertices }\} \mid .
\end{aligned}
$$

This depends only on $C$, not $v_{0}$ or $v_{1}$.

Now choose $T$ in $\mathscr{T}$ and consider the contribution made to $f_{G}(x)$ by those elements in $G$ whose minimal walks end at $v_{0}$, which have value 1 at $v_{0}$ and which have support $T$. Set $f(x)=f_{K}(x)$. Recall Theorem 1.2. Suppose that $T$ has $i$ nonextreme vertices and $j$ nontrivial extreme vertices. The value of 
such a walk at every nonextreme vertex of $T$ can be any element of $K$. Thus $f(x)^{i}$ is a factor of the power series in question. The value of such a walk at every nontrivial extreme vertex of $T$ can be any nontrivial element of $K$. Thus $(f(x)-1)^{j}$ is also a factor of the power series in question. Because the Euler characteristic of $T$ is $1, T$ has $i+j$ edges. Thus the length of such a walk is $2(i+j)$, and so $x^{2(i+j)}$ is also a factor. Therefore the contribution made to $f_{G}(x)$ by those elements in $G$ whose minimal walks end at $v_{0}$, which have value 1 at $v_{0}$ and which have support $T$ is $\left(x^{2} f(x)\right)^{i}\left(x^{2}(f(x)-1)\right)^{j}$.

It follows that the contribution made to $f_{G}(x)$ by those elements in $G$ whose minimal walks end at $v_{0}$, which have value 1 at $v_{0}$ and which have support in $\mathscr{T}$ is $F_{C}\left(x^{2} f(x), x^{2}(f(x)-1)\right)$. Set $F(x)=F_{C}\left(x^{2} f(x), x^{2}(f(x)-1)\right)$.

Now choose a vertex $v \neq v_{0}$ in $C$, and consider the contribution made to $f_{G}(x)$ by those elements in $G$ whose minimal walks end at $v$. Such a walk begins at $v_{0}$, and the following may be assumed.

$$
\begin{aligned}
& \text { It first goes a certain amount in the } m-1 \text { directions away from } \\
& v \text {. }
\end{aligned}
$$

Then it goes toward $v$. At each interior vertex of the geodesic from $v_{0}$ to $v$ it goes a certain amount in the $m-2$ directions away from the geodesic.

The parts of these walks described in (2.2) contribute a factor of $F(x)$ to the power series in question for each of the $m-1$ directions away from $v$. They also contribute a factor of $f(x)$ which arises from the values of the walks at $v_{0}$. Thus the parts of these walks described in (2.2) contribute a factor of $f(x) F(x)^{m-1}$ to the power series in question. In the same way the parts of these walks described in (2.3) contribute a factor of $\left(f(x) F(x)^{m-2}\right)^{d\left(v_{0}, v\right)-1}$, where $d$ is the standard metric on $C$. Just as for (2.2), the factor corresponding to (2.4) is $f(x) F(x)^{m-1}$. Finally, the distance travelled along the geodesic from $v_{0}$ to $v$ is taken into account by the factor $x^{d\left(v_{0}, v\right)}$. Thus the contribution made to $f_{G}(x)$ by those elements in $G$ whose minimal walks end at $v$ is

$$
f(x) F(x)^{m}\left(x f(x) F(x)^{m-2}\right)^{d\left(v_{0}, v\right)} .
$$

The above is also correct if $v=v_{0}$, and so

$$
\begin{aligned}
f_{G}(x) & =\sum_{v \in \operatorname{vert}(C)} f(x) F(x)^{m}\left(x f(x) F(x)^{m-2}\right)^{d\left(v_{0}, v\right)} \\
& =f(x) F(x)^{m} f_{H}\left(x f(x) F(x)^{m-2}\right) .
\end{aligned}
$$

Because $C$ is a tree every vertex of which has valance $m$, it is not difficult to see that

$$
f_{H}(x)=\frac{1+x}{1-(m-1) x} .
$$

This proves the following theorem. 
Theorem 2.6. Suppose that $C$ is a tree every vertex of which has valence $m$. Let $f(x)=f_{K}(x)$, and let $F(x)=F_{C}\left(x^{2} f(x), x^{2}(f(x)-1)\right)$, where $F_{C}(x, y)$ is defined in line (2.1). Then

$$
f_{G}(x)=\frac{f(x) F(x)^{m}\left(1+x f(x) F(x)^{m-2}\right)}{1-(m-1) x f(x) F(x)^{m-2}} .
$$

\section{Rationality of $f_{G}(x)$}

The assumptions and notation of $\S 2$ remain in force.

Lemma 3.1. The power series $F_{C}(x, y)$ satisfies the following equation for $m \geq 1$.

$$
F_{C}(x, y)=1+y-x+x F_{C}(x, y)^{m-1} .
$$

Proof. Recall the definition of $F_{C}(x, y)$ from line $(2.1)$. The tree $T_{0}$ in $\mathscr{T}$ consisting of just the vertex $v_{0}$ gives the constant term 1 in $F_{C}(x, y)$. The tree $T_{1}$ in $\mathscr{T}$ consisting of the vertices $v_{0}, v_{1}$ and the edge between them contributes $y$ to $F_{C}(x, y)$. Now let $\mathscr{T}^{\prime}$ be the set of subtrees of $C$ gotten by deleting $v_{0}$ and the edge between $v_{0}$ and $v_{1}$ from the trees in $\mathscr{T}-\left\{T_{0}\right\}$. Then $\mathscr{T}^{\prime}$ is the set of all finite subtrees of $C$ which contain $v_{1}$ but not $v_{0}$. It is easy to see that the generating function analogous to $F_{C}(x, y)$ for $\mathscr{T}^{\prime}$ is $F_{C}(x, y)^{m-1}$. Thus $\mathscr{T}-\left\{T_{0}, T_{1}\right\}$ contributes $x\left(F_{C}(x, y)^{m-1}-1\right)$ to $F_{C}(x, y)$. Combining the above proves the lemma.

Corollary 3.2. The power series $F(x)$ satisfies the following equation for $m \geq 1$.

$$
F(x)=1-x^{2}+x^{2} f(x) F(x)^{m-1} .
$$

Proof. This is easy to verify using Lemma 3.1 .

When $m=1,2$ this result can be used to express $F(x)$ as a rational function in $x$ and $f(x)$, which together with Theorem 2.6 proves the following.

\section{Corollary 3.3.}

$$
f_{G}(x)= \begin{cases}f(x), & m=0 \\ (1+x) f(x)(1-x+x f(x)), & m=1 \\ \frac{f(x)\left(1-x^{2}\right)^{2}(1+x f(x))}{\left(1-x^{2} f(x)\right)^{2}(1-x f(x))}, & m=2 .\end{cases}
$$

In particular, if $f(x)$ is rational in these cases, then so is $f_{G}(x)$.

Corollary 3.4. Suppose that $m \geq 1$, and assume the hypotheses of Theorem 2.6. Then

$$
f_{G}(x)=\frac{f(x) F(x)^{m}\left(1+x f(x) F(x)^{m-2}\right)}{1-(m-1) x f(x) F(x)^{m-2}},
$$

where

$$
F(x)=1-x^{2}+x^{2} f(x) F(x)^{m-1} .
$$

Proof. This merely combines Theorem 2.6 and Corollary 3.2.

A straightforward computation shows that this result transforms into the following one by means of the substitution $E(x)=\left(1-x^{2}\right) / F(x)$. 
Corollary 3.5. Suppose that $m \geq 1$, and assume the hypotheses of Theorem 2.6. Then

$$
f_{G}(x)=\frac{\left(1-x^{2}\right)^{2}(E(x)-1)(E(x)-x-1)}{x^{2} E(x)^{2}((m-1) E(x)+x+1-m)},
$$

where

$$
E(x)^{m-1}-E(x)^{m-2}+x^{2}\left(1-x^{2}\right)^{m-2} f(x)=0 .
$$

The rest of this section deals with the case $m>2$.

Lemma 3.6. Suppose that $m>2$ and that $K \neq 1$, namely, $f(x) \neq 1$. Then $F(x)$ is not in the field generated over $\mathbb{Q}$ by $x$ and $f(x)$.

Proof. It is clear that $f(x)$ and $F(x)$ are power series in $x$ with nonnegative integer coefficients.

Let $R_{1}$ be the radius of convergence of $F(x)$, and let $R_{2}$ be the radius of convergence of $f(x)$. Since $F(x)$ has infinitely many nonzero coefficients, $R_{1} \leq 1$. (Since the coefficients of $F(x)$ are dominated by the coefficients of $f_{G}(x)$ and the radius of convergence of $f_{G}(x)$ is positive, $R_{1}>0$.) The definition of $F(x)$ easily shows that $R_{1} \leq R_{2}$.

It will now be shown that if the nonnegative real number $r$ increases to $R_{1}$, then $F(r)$ increases to a finite limit. Indeed, the above shows that $F(r)$ and $f(r)$ are positive increasing functions of the real number $r$ for $0 \leq r<R_{1}$. If $F(r)$ increases to $\infty$ as $r$ increases to $R_{1}$, then Corollary 3.2 shows that $F(r)$ increases to $\infty$ with at least the order of magnitude of $F(r)^{m-1}$. This is impossible. Thus $F(r)$ increases to a finite limit as $r$ increases to $R_{1}$.

This combined with line $(0.1)$ and Corollary 3.2 implies that $R_{1}<R_{2}$. Therefore if $F(x)$ is in the field generated over $\mathbb{Q}$ by $x$ and $f(x)$, then $R_{1}$ is a pole of $F(x)$, contrary to the fact that $F(r)$ increases to a finite limit as $r$ increases to $R_{1}$. This proves Lemma 3.6.

The next result is the analog of Corollary 3.3 for the case in which $m>2$.

Theorem 3.7. Suppose that $m>2$ and $K \neq 1$. Then $f_{G}(x)$ is algebraic over the field generated over $\mathbb{Q}$ by $x$ and $f(x)$, but it is not contained in this field.

Proof. Let $L$ be the field generated over $\mathbb{Q}$ by $x$ and $f(x)$. Corollary 3.5 shows that $f_{G}(x)$ is algebraic over $L$. Thus what must be shown is that $f_{G}(x)$ is not contained in $L$. The following paragraph of commutative algebra prepares for this.

Corollary 3.5 shows that $E(x)$ is algebraic over $L$, and Lemma 3.6 shows that $E(x)$ is not contained in $L$. Let $M$ be the splitting field of the irreducible polynomial of $E(x)$ over $L$. Let $A$ be the subring of $L$ consisting of those elements in $L$ which are holomorphic at 0 . Let $B$ be the integral closure of $A$ in $M$. The evaluation map $x \mapsto 0$ induces a ring homomorphism $\varphi: A \rightarrow \mathbb{C}$. In particular, $\varphi$ restricted to $\mathbb{Q}$ is the identity map, $\varphi(x)=0$ and $\varphi(f(x))=$ 1. A standard result in commutative algebra (see Chapter V, $\S 2.1$, Corollary 4 of [1]) states that $\varphi$ can be extended to a ring homomorphism, still denoted by $\varphi$, from $B$ to $\mathbb{C}$. Let $E_{1}(x), \ldots, E_{n}(x)$ denote the algebraic conjugates of $E(x)$ over $L$. They lie in $B$ because Corollary 3.5 shows that they are roots of the monic polynomial

$$
P(y)=y^{m-1}-y^{m-2}+x^{2}\left(1-x^{2}\right)^{m-2} f(x)
$$


which lies in the polynomial ring $A[y]$. There is a unique further extension of $\varphi$ to a ring homomorphism $\varphi: B[y] \rightarrow \mathbb{C}[y]$ which maps $y$ to $y$. Clearly,

$$
\varphi(P(y))=y^{m-1}-y^{m-2},
$$

and this is divisible by

$$
\varphi\left(\prod_{i=1}^{n}\left(y-E_{i}(x)\right)\right)=\prod_{i=1}^{n}\left(y-\varphi\left(E_{i}(x)\right)\right) .
$$

Thus at most one of the values $\varphi\left(E_{1}(x)\right), \ldots, \varphi\left(E_{n}(x)\right)$ is 1 , and the others are 0 . The Galois group of $M$ over $L$ acts on the set $\left\{E_{1}(x), \ldots, E_{n}(x)\right\}$ transitively. By composing $\varphi$ with a field automorphism of $M$ over $L$, it may therefore be assumed that $\varphi(E(x))=0$.

Now consider $\varphi$ and $f_{G}(x)$. The value of $\varphi$ at the numerator of the right side of the first equation in Corollary 3.5 is 1 , and the value of $\varphi$ at this denominator is 0 . Thus $f_{G}(x)$ is not in $B$, hence it is not in $A$ and so it is not in $L$. This proves Theorem 3.7.

Corollary 3.8. If $m>2$ and $K \neq 1$, then $f_{G}(x)$ is not a rational function.

\section{RADIUS OF CONVERGENCE OF $f_{G}(x)$}

In addition to the assumptions and notation of $\S 2$, in this section it will be assumed that $m \geq 2$. Here is the main result of this section.

Theorem 4.1. If $m \geq 2$, then the radius of convergence of $f_{G}(x)$ is the smallest positive zero of

$$
\left(1-\frac{x}{m-1}\right)^{m-2}-(m-1) x\left(1-x^{2}\right)^{m-2} f(x) .
$$

Proof. Let $R$ be the radius of convergence of $f_{G}(x)$. As in the second paragraph of the proof of Lemma 3.6, the radius of convergence of $f(x)$ is at least the radius of convergence of $F(x)$ and $R$ is at most the radius of convergence of $F(x)$. Since $f(r)$ and $F(r)$ are positive increasing functions of $r$, line $(0.1)$ and the first equation in Corollary 3.4 show that $R$ is the smallest positive zero of $1-(m-1) x f(x) F(x)^{m-2}$. Using the second equation in Corollary 3.4 and the fact that $E(x)=\left(1-x^{2}\right) / F(x)$, it is then a routine matter to see that $R$ is the smallest positive solution of the equation $E(x)=1-x /(m-1)$.

This and the second equation in Corollary 3.5 lead to the polynomial

$$
P(y)=y^{m-1}-y^{m-2}+t,
$$

where $t$ is a real number. Let $\alpha_{1}(t), \ldots, \alpha_{m-1}(t)$ be generically distinct roots of $P(y)$ which vary continuously with $t$. Assume that $\alpha_{1}(0)=1$, and so

$$
\alpha_{2}(0)=\cdots=\alpha_{m-1}(0)=0 .
$$

Because the zeros of $P^{\prime}(y)$ lie in the set $\{0,(m-2) /(m-1)\}$, it is not difficult to prove the following. If $i$ is an integer with $1 \leq i \leq m-1$ and $t$ is a real number such that $\alpha_{i}(t)$ lies in the open interval $((m-2) /(m-1), 1)$, then $i=1$. Furthermore, if $\alpha_{i}(t)=(m-2) /(m-1)$, then $\alpha_{1}(t)=(m-2) /(m-1)$.

Now combine the previous two paragraphs. Since $1-r /(m-1)$ varies from 1 to $(m-2) /(m-1)$ as $r$ varies from 0 to 1 , it follows that $E(R)$ lies in 
the interval $[(m-2) /(m-1), 1)$. Thus the previous paragraph and the second equation in Corollary 3.5 imply that $R$ is the smallest positive zero of

$$
\left(1-\frac{x}{m-1}\right)^{m-1}-\left(1-\frac{x}{m-1}\right)^{m-2}+x^{2}\left(1-x^{2}\right)^{m-2} f(x) \text {. }
$$

Factoring $(1-x /(m-1))^{m-2}$ from the first two terms of this expression allows it to be slightly simplified to give the desired result. This proves Theoremi 4.1.

\section{EXAMPLES}

Example 5.1. Take $m=2$. By Corollary 3.3

$$
f_{G}(x)=\frac{f(x)\left(1-x^{2}\right)^{2}(1+x f(x))}{\left(1-x^{2} f(x)\right)^{2}(1-x f(x))},
$$

and by Theorem 4.1 the radius of convergence of $f_{G}(x)$ is the smallest positive zero of $1-x f(x)$.

It is clear that the largest radius of convergence for $f_{G}(x)$ with nontrivial $K$ occurs for $K=\mathbb{Z} / 2 \mathbb{Z}$. In this case $f(x)=1+x$, from which it easily follows that the radius of convergence of $f_{G}(x)$ is $(\sqrt{5}-1) / 2$. Line $(2.5)$ easily implies that this is the largest radius of convergence for all pairs $\left(G, S_{G}\right)$ with $m \geq 2$ and $K \neq 1$ if $m=2$.

Taking $H=\mathbb{Z}$ and $K=\mathbb{Z}^{2}$ gives the "super-group" from Chapter 4 of Grayson's thesis [3]. More generally, taking $H=\mathbb{Z}$ and $K=\mathbb{Z}^{r}$ gives a group $G$ which maps onto all torus bundle groups $\mathbb{Z}^{r} \rtimes \mathbb{Z}$ of rank $r$.

Example 5.2. Take $m=3$ and $K=\mathbb{Z} / 2 \mathbb{Z}$, so that $f(x)=1+x$. Corollary 3.4 and Theorem 3.7 show that $f_{G}(x)$ is a root of an irreducible quadratic polynomial over the rational function field $\mathbb{Q}(x)$. Theorem 4.1 shows that the radius of convergence of $f_{G}(x)$ is the smallest positive root of $1-x / 2-$ $2 x\left(1-x^{2}\right)(1+x)$. This radius of convergence is approximately .3485 . This polynomial is in fact irreducible over $\mathbb{Q}$. It easily follows that the reciprocal of this radius of convergence is an algebraic number but not an algebraic integer, further evidence that $f_{G}(x)$ is irrational.

\section{REFERENCES}

1. N. Bourbaki, Commutative algebra, Addison-Wesley, Reading, Mass., 1972.

2. J. Cannon, The growth of the closed surface groups and the compact hyperbolic groups, preprint.

3. M. Grayson, Geometry and growth in three dimensions, Thesis, Princeton Univ., Princeton, N. J., 1983.

4. R. Grigorchuk, Degrees of growth of finitely generated groups, and the theory of invariant means, Izv. Akad. Nauk SSSR 48 (1984).

5. J. Milnor, A note on curvature and fundamental group, J. Differential Geom. 2 (1968), 1-7.

6. J.-P. Serre, Trees, Springer-Verlag, New York, 1980.

Department of Mathematics, Eastern Michigan University, Ypsilanti, Michigan 48197 